\title{
La guerre des ondes entre la France et l'Allemagne pendant la « drôle de guerre »
}

\author{
MAUdE FAGOT
}

À partir de l'Entre-deux-guerres, la radiodiffusion constitue l'un des médias modernes de masse par excellence. Son rôle primordial dans les sociétés occidentales a été maintes fois étudié, tant dans une perspective d'analyse sociologique et médiatique ${ }^{1}$, qu'historique. Les historiens français, britanniques, allemands, mais également américains se sont pris d'intérêt pour ce sujet et notamment pour son développement pendant la Seconde Guerre mondiale. Ils ont apporté des perspectives nationales, des thématiques et des méthodologiques différentes, permettant de dresser un portrait pluriel de cette «guerre des ondes ». Parmi les historiens qui se sont penchés sur les propagandes radiophoniques européennes pendant le second conflit mondial, certains ont fait porter leurs analyses sur les institutions de radiodiffusion et leurs fonctionnements ${ }^{2}$, et plus rarement, sur la question de la réception et des effets des émissions sur la population en temps de guerre ${ }^{3}$. D'autres ont pris le parti d'étudier une station radiophonique en particulier, c'est le cas par exemple du travail de Nelson Costa Ribeiro portant sur le rôle joué par les émissions de la BBC au Portugal $^{4}$, ou bien de l'ouvrage de David O'Donoghue sur une des émission allemandes dirigée vers l'Irlande 5 . Loin d'être un domaine délaissé de la recherche historique, l'étude de la propagande radiophonique pendant la Seconde Guerre mondiale en Europe recèle encore certaines zones d'ombre. C'est notamment le cas pour le conflit franco-allemand pendant la « drôle de guerre ».

En France, le regard du chercheur s'est tourné prioritairement vers la période de l'Occupation ${ }^{6}$ et vers la radiodiffusion de la France Libre ${ }^{7}$, tandis qu'en Allemagne, l'intérêt a été porté particulièrement aux institutions et à quelques émissions ${ }^{8}$. Certains ouvrages mentionnent cependant l'action de la propagande radiophonique française et allemande, mais ne s'y consacre pas entièrement pour autant ${ }^{9}$. De plus, bien que les radios

\footnotetext{
1 Cécile Méadel, Publics et mesures, une sociologie de la radio, rapport CNI-CNRS, École des mines de Paris, 1986, Beno Sternberg, Aspects sociaux de la radio et de la télévision, Confluence, vol. IV, Paris, 1996 ; Marshall McLuhan, The Medium is the Massage: An Inventory of Effects, New York, Random House, 1967

2 Philippe Amaury, Les Deux premières expériences d'un Ministère de l'information en France: L'apparition d'institutions politiques et administratives d'information et de propagande sous la IIIe République en temps de crise juillet 1939- juin 1940, leur renouvellement par le régime de Vichy juillet 1940 - août 1944, Paris, Librairie générale de droit et de jurisprudence, 1969 ; Diller Angsar, Rundfunkpolitik im Dritten Reich, dtv, München, 1980

3 Diller Angsar, op.cit.; Martin A. Doherty, Nazi wireless propaganda: Lord Haw-Haw and British public opinion in the Second World War, Edinburgh, Edinburgh Univ. Press, 2000.

4 Nelson Costa Riberio, BBC broadcasts to Portugal in World War II : how radio was used as a weapon of war, Lewiston, Mellen, 2011.

5 David O'Donoghue, Hitler's Irish voices : the story of German Radio's wartime Irish service, Belfast, Beyond the Pale Publ., 1998.

6 Agnès Callu, Patrick Eveno, Hervé Joly (dir.), Culture et médias sous l’Occupation, Paris, Éd. du CTHS, 2009.

7Aurélie Luneau, Radio Londres : les voix de la liberté (1940 - 1944), Paris, Perrin, 2005.

8 John A. Cole, Hier spricht der Großdeutsche Rundfunk : Der Fall Lord Haw-Haw, Wien, Zsolnay, 1965.

9 Le seul ouvrage se consacrant entièrement à une émission allemande destinée à la France et celui des historiens Ortwin Buchbender, Reinhard Hauschild, Geheimsender gegen Frankreich: die Täuschungsoperation «Radio Humanité »1940, Herford, Mittler, 1984. Dans quelques chapitres des ouvrages suivants, la propagande radiophonique française est thématisée, sans malheureusement faire l'objet d'une analyse approfondie : Hélène Eck (dir.), La Guerre des ondes : histoire des radios de langue française
} 
françaises et allemandes se soient affrontées et aient interagi pendant ces huit mois et demi de drôle guerre, aucune étude historique jusqu'à ce jour ne les mets réellement en relation pour tenter de comprendre la variété des informations échangées de la déclaration de guerre au déclanchement de l'offensive allemande en mai 1940.

$\mathrm{Au}$ cours de l'Entre-deux-guerres, la radiodiffusion prend son essor à la fois en France et en Allemagne. Soutien fort pour les dirigeants nazis en Allemagne, elle est également utilisée en France dans les années 1930, pour diffuser les discours des hommes politiques. Par le biais de ce média, l'information sort de son cadre national et dépasse aisément les frontières. De plus, la naissance d'émissions de radio en langue étrangère ouvre de nouvelles perspectives en matière de diffusion de l'information et notamment de propagande à l'étranger. En France, Radio Strasbourg diffuse à l'approche du plébiscite pour le rattachement de la Sarre au Reich (1934-1935) une forte campagne anti-allemande en langue allemande, qui se trouve être à l'origine de nombreuses tensions entre les autorités diplomatiques franco-allemandes ${ }^{10}$. Au cours de l'année 1936, l'impact de la radio française sur l'opinion publique effraie les autorités allemandes et elles pensent déjà aux risques de son utilisation en cas de conflit:

\footnotetext{
«Les expériences de la période de tension du mois de mars [il s'agit de la remilitarisation de la Rhénanie en 1936] peuvent se résumer ainsi : la technique de radiodiffusion française est une arme excellente pour mener une guerre psychologique, qui est tactiquement si intelligemment menée, que dans chaque cas de tension de politique extérieure, elle joue un rôle essentiel dans l'influence de l'opinion publique mondiale $»^{11}$.
}

Des institutions visant à mettre sous la tutelle de l'Etat la diffusion d'émissions en direction de l'ennemi se mettent en place au courant de l'année 1939 des deux côtés de la frontière. Elles entrent en action, plus ou mois tôt pendant la «drôle de guerre » et sont utilisées de manière accrue au fil des mois.

Bien que certains historiens ${ }^{12}$ affirment que ce combat sur les ondes s'est fait de manière inégale au désavantage de la France, l'étude des sources françaises et allemandes tend à relativiser ce point de vue ${ }^{13}$. On distingue deux sortes d'émissions, aussi bien en France qu'en Allemagne : les « radios blanches », qui affichent clairement leur émetteur et les « radios noires » dont le but est de faire croire à une radio nationale. Au travers de ces différentes émissions, une propagande de démoralisation de l'armée, mais également de la population voit le jour. Afin d'atteindre ce but, les Etats développent des thématiques adaptées à la situation politique, économique et sociale de l'adversaire.

\section{LA MISE EN PLACE D'UNE PROPAGANDE RADIOPHONIQUE EXTERIEURE}

\section{En Allemagne}

Pendant la période de la «drôle de guerre », quelques changements s'opèrent dans l'organisation de la radio en langues étrangères en Allemagne. Au centre des querelles

pendant la Deuxième guerre mondiale, Paris, A. Colin, 1985 ; Jean-Louis Crémieux-Brilhac, Les Français de l'an 40, t.1, La guerre, oui ou non ?, Paris, Gallimard-NRF, 1990.

10 L'ambassadeur allemand à Paris se plaignait auprès du ministre des Affaires étrangères, Pierre Laval, d'une « constante augmentation de la haine de l'émetteur de Strasbourg à l'encontre de l'Allemagne », cité dans : Angsar Diller, Rundfunkpolitik im Dritten Reich, München, Deutscher Taschenbuch Verlag, 1980, p. 240.

11 Ibidem, p. 244.

12 Philippe Amaury, op.cit. ; Henri Amouroux, Le peuple du désastre 1939-1940, Paris, R. Laffont, 1976.

13 Pour ce travail nous nous appuyons sur des rapports militaires français et allemands, ainsi que des sources des ministères responsables de la propagande des deux pays. 
entre le ministre des Affaire étrangère Joachim von Ribbentrop et le ministre de la propagande Joseph Goebbels, la radiodiffusion subit des remaniements. Le Führer Befehl du 8 septembre 1939 remet en cause la suprématie du ministère de la propagande dans le domaine des émissions radiophoniques pour l'étranger, suprématie qu'il avait obtenu quelques années plus tôt avec la loi du 10 juin $1933^{14}$, au détriment du ministre des Affaires étrangères de l'époque, Konstantin von Neurath. Cependant, Goebbels n'accepte pas la suppression de ses prérogatives. Il concède à laisser entrer le personnel de Ribbentrop dans l'organisation du Auslandsnachrichten Dienst, mais ne permit jamais l'intégration de ce personnel dans le système de diffusion (Funkverbindungsstelle) ${ }^{15}$. Bien que ces querelles aient pu compliquer le fonctionnement de la radiodiffusion à l'étranger, il est essentiel de constater que les radios fonctionnent sans interruption jusqu'à la signature de l'armistice le 22 juin 1940.

Un organigramme ${ }^{16}$ permet de visualiser la multitude des services qui étaient impliqués dans la radiodiffusion à l'étranger, notamment le ministère des Affaires étrangères, le ministère de la propagande et sous son contrôle, la Reichsrundfunkgesellschaft, fournissant les moyens financiers d'action. Les volontés d'innovation, notamment celles du Dr. Adolf Heinrich Raskin, à la tête du Büro Concordia constituent un élément clé pour le développement des radios à l'étranger. Directeur de l'émetteur de Sarrebruck pendant l'Entre-deux-guerres et donc très au fait des problèmes liés à la radiodiffusion étrangère, il accumule au cours de la «drôle de guerre » les responsabilités de directeur de l'Auslandsabteilung der Reich-Rundfunk-Gesellschaft puis d'intendant de la section Deutsche Kurzwellensender ${ }^{17}$. Il est convaincu de la puissance de la radio et de la propagande. Dans son article «Dramaturgie und Propaganda », il écrit :

«L'effet le plus notoire de la radio vient des mots. [...] Propagande signifie élargir, diffuser, soutenir, venter les mérites d'idées et des connaissances, combattre sur tous les fronts de l'esprit, fertiliser, propager, exterminer, dissuader et éradiquer, construire et détruire. Dans le mot «propagande » se trouve à la fois la direction et l'objectif ${ }^{18}$.

C'est avec ces idées qu'il dirige la section Concordia chargée des émissions secrètes en langues étrangères à destination des pays ennemis ou neutres. Peu d'archives ont été préservées sur cette section, réduisant ainsi les possibilités de reconstruction des prérogatives, des structures et des travaux effectués par le Büro Concordia. Cependant, certaines sources à la fois françaises et allemandes sur le contenu des émissions permettent de dresser un tableau des thématiques de propagande alors employées par les services allemands ${ }^{19}$.

Parallèlement et en collaboration avec les institutions de propagande civile, l'armée joue un rôle important pour la diffusion et l'étude de la réception des émissions radiophoniques à destination de la France. Les Propaganda-Kompanien ${ }^{20}$ créées dans le

14 Verordnung über die Aufgaben des Reichsministeriums für Volksaufklärung und Propaganda (30.06.1933), http://www.documentArchiv.de/ns/propaganda_vo.html Site consulté en octobre 2013.

15 Horst Bergmeier, Rainer Lotz, Hitler's Airwaves: The Inside Story of Nazi Radio Broadcasting and Propaganda Swing, Yale, Yale University Press, 1997, p. 181.

16 Voir Annexe 1

17 Horst Bergmeier, Rainer Lotz, op.cit., p. 182.

18 Traduit par l'auteur de Ortwin Buchbender, Reinhard Hauschild, Geheimsender gegen Frankreich: die Täuschungsoperation « Radio Humanité »1940, Herford, Mittler, 1984, p. 41.

19 Les transcriptions des émissions de Radio Humanité se trouvent aux archives politiques du ministère des Affaires étrangères allemand. Celles de Radio Stuttgart sont conservées aux archives nationales à Paris.

20 L'accord entre OKW et le ministère de Goebbels, «Abkommen über die Durchführung der Propaganda im Kriege » signé pendant l'hiver 1938/9 prévoit qu'en cas de guerre une « guerre psychologique » serait menée, dirigée par le ministère de la propagande. En avril 1939, Oberkommando Wehrmacht/ Wehrmacht Propaganda (OKW/WPr) et avec lui, les Propaganda Kompanien (PK) deviennent une structure fixe. 
but de mener des campagnes de propagande au front, diffusent des émissions radiophoniques par le biais d'émetteurs «mobiles ${ }^{21}$ dont celui installé le 18 novembre 1939 à Schopfheim en Bade. Il avait diverses fonctions : brouiller certains émetteurs (Störsender), diffuser des émissions à l'intention de l'ennemi (Propaganda-Kampf Sender), mais aussi des émissions de soutien moral pour les troupes allemandes (Soldatensender zur Betreuung der eigenen Soldaten) ${ }^{22}$. Le rôle majeur des compagnies de propagande dans le domaine de la radio consiste à rendre compte des contenus des radios étrangères, à travers leur collaboration avec les Abteilung Ic (Feindaufklärung und Abwehr, geistige Betreuung) de chaque division. L'armée fournit ainsi au RMVP par le biais de Lageberichte der Rundfunk ${ }^{23}$ de nombreux renseignements sur le contenu des émissions françaises en langue allemande (Radio Strasbourg en particulier) qui constituent des sources particulièrement intéressantes pour l'historien.

\section{En France}

Bien qu'en France, la mise au pas de la radiodiffusion se soit effectuée plus tardivement, il n'est pas possible d'affirmer que le gouvernement français ait pu set laisser prendre au dépourvu. Depuis 1938 s'effectuent en France certaines modifications tendant vers une centralisation et un contrôle étatique de la radiodiffusion ${ }^{24}$. Daladier crée le Réseau de Radiodiffusion National en juillet 1939, couplé avec la création du Commissariat Général à l'Information. Avec à sa tête l'écrivain Jean Giraudoux, ce commissariat placé directement sous la tutelle du Président du Conseil a pour but «d'organiser, d'animer et de coordonner tous les services d'information et d'expansion française ${ }^{25}$. Dans les faits, la collaboration entre le Commissariat et la Radiodiffusion est difficile. La section des émissions en langues étrangères appartient directement au Centre permanent de l'Information générale dirigée par $M$. Lohner. Celui-ci est relié au Commissariat par l'intermédiaire de Jacques Fouques-Duparc, haut fonctionnaire des Affaires étrangères et responsable au sein du Commissariat de Giraudoux de contrôler les émissions radiophoniques pour l'étranger. C'est également à travers ce « super directeur politique des émissions étrangères ${ }^{26}$ que le Quai d'Orsay garde une influence sur les émissions à l'étranger en lui faisant parvenir ses consignes. Au sein du Commissariat Général à l'Information, est créé un Service de l'Information pour l'Etranger (SIE). Il contient une section Allemagne dans laquelle travaillent notamment $\mathrm{M}$. Tonnelat et $\mathrm{M}$. Vermeil, tous deux professeurs d'allemand à l'Université, M. Tonnelat ayant déjà participé aux campagnes de propagande de la Première Guerre mondiale. Le rôle et l'action de cette section sont défini comme suit dans un rapport du Commissaire Général Jean Giraudoux :

«La Section Allemagne a des relations régulières avec le service des Emissions en langue allemande. Elle lui fournit des suggestions et examine les émissions en question. Elle participe à ces émissions de manière directe, pour l'instant (4 janvier 1940) à raison de 1 par semaine $»^{27}$.

L'enchevêtrement de ces institutions n'est pas sans rappeler les problèmes auxquels sont confrontés les autorités allemandes et engendre, tout comme en Allemagne, des problèmes de communication et des rivalités entre les services. En guise d'exemple, on

21 Ortwin Buchbender, Reinhard Hauschild, op.cit., p. 31.

22 Idem.

23 Bundesarchiv/Militärarchiv (désormais BArch-MA), RW4/242.

24 Philippe Amaury, op.cit., p.398-399.

$25 \mathrm{Idem}$.

26 Hélène Eck, op.cit., p. 20.

27 Archives Nationales (désormais AN) F41/19-20. 
mentionnera une note du 29 octobre 1939 établie par le Service d'Information à l'Etranger signalant que de nombreux textes rédigés par M. Tonnelat et $\mathrm{M}$. Vermeil n'avaient pas été utilisés par les services de $\mathrm{M}$. Copeau, responsable de la diffusion des émissions radiophoniques en langue allemande ${ }^{28}$. Dans ce labyrinthe hiérarchique, ces émissions contre l'ennemi occupent une place particulièrement importante.

Bien qu'elles aient fait couler beaucoup d'encre à l'époque entre les différents services et qu'elles soient mentionnées dans de nombreux ouvrages, il nous manque des informations précises sur la manière d'opérer de ces émissions en langue allemande. Il a cependant été possible de reconstituer une grande partie de leur organisation en s'appuyant sur des biographies, des archives du Commissariat Général à l'Information et des courriers entre les différents services, conservés aux Archives Nationales à Paris ${ }^{29}$. Comme nous l'avons fait remarquer plus haut, les émissions en langues étrangères mêlent différents acteurs. Ceci est d'autant plus marqué pour ce qui est des émissions en langue allemande. En effet, parallèlement à l'intervention du CGI et du Quai d'Orsay, le Grand Quartier Général compte bien faire entendre sa voix au travers de sa Section de Propagande contre l'Ennemi.

Tous tentent alors de jouer d'influence sur le travail dirigé par Pascal Copeau, chef de service des ondes moyennes (c'est-à-dire en direction de l'Europe). Cet homme n'est pas un novice dans le milieu de la radiodiffusion contre l'Allemagne. Depuis 1938 il est responsable des émissions en langue allemande de Radio Strasbourg et dirige à partir de l'évacuation de l'émetteur de Strasbourg les émissions nationales en langue allemande depuis Paris ${ }^{30}$. Il est assisté dans sa tâche à partir d'avril 1940 par Pierre Bertaux, un germaniste qui avait quitté la radio et les émissions en langue allemande en 1938 pour prendre une chaire de littérature allemande à Toulouse, puis qui avait, à la mobilisation, servi en tant que traducteur au IIème Bureau militaire du G.Q.G ${ }^{31}$. Ces deux dirigeants, experts en propagande contre le Reich, travaillent en collaboration avec des traducteurs et des speakers, le plus souvent immigrés politiques allemands. Bien qu'au moment de la déclaration de guerre, ils aient été retenus dans des «centres de rassemblement », ils sont toutefois libérés et réintégrés après quelques jours pour pouvoir participer à la " guerre des ondes $»^{32}$. Cette détention en centre de rassemblement fait ralentir quelque peu la radio en langue allemande, mais n'est en rien comparable avec ce qu'il se passe au mois de mai 1940. En effet, lorsque l'armée allemande entre en territoire français, les speakers réfugiés allemands sont arrêtés par la police et les explications de Pierre Bertaux ne suffisent pas à assurer leur liberté pour continuer à diffuser ${ }^{33}$. Parmi eux on compte entre autres : Alexander Mass Werth, Hans Jacob, ancien interprète de Stresemann, le juriste Wolf Franck, Max Ophüls, cinéaste allemand, Hans Siemsen, romancier homosexuel dont les œuvres ont été brûlées en place publique par les nazis, la comédienne Anne-Marie Seekel et l'acteur Karl Heil, connu plus tard sous le nom de Charles Hébert ${ }^{34}$. À ceux-ci s'ajoutent

28 AN/F41/21-22, « Note sur la radio française », 29.10.1939.

29 Voir Annexe 2.

30 Laurent Douzou, «Pascal Copeau » in Marcot (dir.), Dictionnaire historique de la Résistance, Paris, Robert Laffont, 2006 ; Pierre Leenhardt, Pascal Copeau (1908-1982) L'histoire préfère les vainqueurs, Paris, L'Harmattan, 1994.

31 Pierre Bertaux, Mémoires interrompus, Asnières, PIA, 2000, p. 133.

32 Idem.

33 Ibidem, p. 138.

34 Une note du Commissariat Général à l'Information mentionne également les collaborateurs des émissions en langue allemande suivant : M. Dyck, M. Levi, M. Wronkow, M. Handler, M. Schlesinger et M. Lewy, AN $\mathrm{F} / 41 / 21-22$. 
l'actrice française Françoise Rosay ainsi que certains intervenants à titre exceptionnel comme le bâtonnier alsacien Maître Heitz ${ }^{35}$.

\section{LES EMISSIONS RADIOPHONIQUES FRANÇAISES ET ALLEMANDES CONTRE L'ENNEMI}

C'est dans ces deux cadres institutionnels différents mais dont les difficultés sont similaires que trouvent leur place les différentes émissions de radiodiffusion allemandes et françaises en direction de l'ennemi de septembre 1939 à mai 1940. Concernant ces émissions, il est nécessaire de faire une distinction entre les « radios noires », qui mènent une campagne cachée de l'information se faisant passer pour des radios nationales et les « radios blanches », c'est-à-dire celles qui ne cachent pas leurs origines.

\section{Les radios «blanches » : Radio Stuttgart vs. Radio Strasbourg}

L'émission de programmes en langues étrangères en Europe trouve son origine en France avec la création le 11 novembre 1930 de Radio Strasbourg P.T.T. Elle était alors le premier poste bilingue d'Europe, diffusant des émissions en langue allemande et en langue française $^{36}$. Particulièrement dynamique et active, cette radio diffusait des émissions profrançaises, fortement engagées lors du référendum sur le ralliement de la Sarre au Reich en 1935, comme nous l'avons évoqué en introduction. Après l'évacuation d'Alsace du siège de Radio Strasbourg le 13 septembre 1939, l'émetteur de Brumath est alimenté par les émissions venant de Paris. Surnommé Lügensender («émetteur menteur ») par Goebbels, Radio Strasbourg continue son action pendant toute la «drôle de guerre » et sera volontairement réduite au silence par l'armée française lors de l'avancée des troupes allemandes. Les acteurs dont nous avons parlé précédemment travaillent alors tous à Paris afin de transmettre les émissions à Radio Strasbourg. Ces émissions se caractérisent à l'ouverture par le bourdon de la cathédrale de Strasbourg ainsi que par la phrase introductive «Allo Ici Radio Strasbourg - Hier Radio Straßburg » et se clôturent par le chant de la Marseillaise. Aux émissions de théâtre et aux concerts des différents conservatoires d'Alsace et de Lorraine se substituent les retransmissions de Paris P.T.T, notamment Radio Journal, des émissions artistiques et culturelles prévues par Georges Duhamel ainsi que les émissions en langue allemande. Celles-ci doivent constituer une riposte face aux attaques radiophoniques allemandes de Radio Stuttgart et développer des thèmes susceptibles «d'ébranler le moral des Allemands ${ }^{37}$. Elles sont diffusées sur ondes moyennes et font partie des émissions à « grande fréquence », c'est-à-dire émises 5 à 7 fois par jour ${ }^{38}$. Cette radio, au même titre que Radio Stuttgart, peut alors être classée dans la catégorie des « radios blanches », puisqu'elle énonce clairement son émetteur.

En Allemagne, il faut attendre le 20 août 1939 pour qu'une radio allemande, Radio Stuttgart diffuse des émissions en langue française. Celle-ci est fonctionnelle à partir du début de la guerre ${ }^{39}$ et est dirigée par le service à ondes moyennes et courtes (Kurz-Wellen Sender - KWS). Ces émissions sont diffusées depuis quatre émetteurs en Allemagne (Sarrebruck, Stuttgart, Munich, Francfort sur le Main), de manière simultanée ou individuelle. André Obrecht, surnommé «Saint Germain », après avoir été un « acteur de

35 Archives Départementales (désormais AD) Bas-Rhin, 98AL833.

36 Les émissions de la BBC pour l'Europe commencent en 1938. Brooks Tim, British propaganda to France, 1940-1944 machinery, method and message, Edinburgh, Edinburgh University Press, 2007, p.11

37 AN F/41/19-20.

38 AN/F/41/21-22.

39 Certains historiens font remonter son origine à 1937. Horst Bergmeier, op.cit., p. 196. 
dernier ordre $»^{40}$ en France s'adresse chaque jour depuis l'Allemagne aux «auditeurs français ! ${ }^{41}$. Il se fait parvenir les scripts des émissions par la section Der Drahtlose Dienst, section récupérée par la Reichs-Rundfunk-Gesellschaft (RRG) au détriment de la tutelle directe du RMVP fin 1939. Ceux-ci sont rédigés au préalable par Paul Ferdonnet, un ancien journaliste français d'extrême droite et membre de la RRG depuis août 1939. Dès le début de la guerre, Radio Stuttgart émet environs six émissions par jour, dont deux sur ondes courtes, celles de 20h45-20h55 et 22h45-23h00, uniquement à destination exclusive des soldats français de la Ligne Maginot ${ }^{42}$. Le rythme s'accélère ensuite, atteignant neuf émissions journalières audibles à heures fixes ${ }^{43}$ dont celles à destination des troupes françaises. L'objectif de cette radio est de «semer le doute et de détendre les ressorts des énergies françaises, si possible même de préparer l'opinion française à une paix blanche qui laisserait à l'Allemagne ses conquêtes à l'est ${ }^{44}$.

Les radios noires: La Voix de la Paix, Radio Humanité vs. deutsche Freiheitssender, österreichische Freiheitssender

Parallèlement à Radio Stuttgart, les «radio noires » (en allemand Geheimsender) font leurs premiers pas. Celles-ci sont sous la tutelle du Büro Concordia, créé le 12 décembre 1939 par Joseph Goebbels, qui en avait déjà fait la demande le 30 octobre 1939. C'est avec ces idées que, le 16 décembre 1936, il développe au sein du Büro Concordia les radios La Voix de la Paix nommée également Le Réveil de la France, Radio Humanité, puis le 27 décembre, Le Poste du Réveil. La Voix de la Paix et Le Réveil de la France s'avèrent être une seule et même radio qui cependant est nommée de façon différente afin de faire croire à un difficile établissement des radios françaises pour renforcer sa crédibilité aux yeux des auditeurs. Au cours de ces émissions, audibles le soir (18h30, 19h00, 20h30, 21h30, 22h30), des musiques populaires sont diffusées, interrompues brutalement par deux à trois minutes d'informations à caractère humoristique sur les évènements politiques intérieurs français et les relations franco-anglaises. Le programme commence toujours par la marche d'ouverture de l'opéra Carmen et se termine par l'hymne national français ${ }^{45}$. Il est cependant difficile de savoir dans quelle mesure cette radio a été écoutée par la population et l'armée française. Toutefois, un attaché militaire espagnol communique en janvier 1940 aux autorités militaires allemandes que l'on parle énormément de cette radio à Paris, ce qui signifie que son existence est connue sinon de la population, du moins de la sphère politique $^{46}$. Ecoutée par un plus grand nombre et opérationnelle du 16 décembre 1939 au 25 juin $1940^{47}$, Radio Humanité est la « radio noire » en langue française majeure de cette période, prétendant une origine communiste. Son nom est une référence manifeste au journal L'Humanité créé en 1904 par Jean Jaurès et interdit en France le 27 août 1939 suite à la suppression du Parti Communiste Français. Tout comme La Voix de la Paix, Radio Humanité diffuse la même émission cinq fois dans la journée, utilisant la répétition comme moyen d'influence. Chacun de ces programmes commence par l'annonce « Ici parle Radio

40 Jean-Louis Crémieux-Brilhac, op.cit., p. 373.

41 Cette invective marque le début de chaque émission de Radio Stuttgart, AN F/43/95-133.

42 AN F/43/95.

$4312 \mathrm{~h} 45$ à 12h55, 14h15 à 14h25, $17 \mathrm{~h} 15$ à 17h25, $20 \mathrm{~h} 15$ à 10h25, 21h15 à 20h30 (ou 45), 22h45 à 23h00,

0h15 à 0h25, Jean-Louis Crémieux-Brilhac, op.cit., p. 380.

44 Idem.

45 RW4/312a, 25. 1. 1940.

46 BArch-MA, RWA/312a, «Auszug aus Bericht des spanischen Mil. Att. In Paris vom 10.1.1940».

47 Ortwin Buchbender, Reinhard Hauschild, op.cit., p. 8. 
Humanité » et est majoritairement composé d'actualités. On ne sait malheureusement pas quelle est la proportion de musique au sein des programmes, néanmoins il est certain que la Marseillaise, l'Internationale et parfois « La Carmagnole » (l'hymne révolutionnaire de 1789) faisaient partie intégrante des émissions ${ }^{48}$. Rédigés à Berlin, les scripts étaient ensuite enregistrés puis diffusés depuis différents émetteurs: Schopfheim en Bade, Cologne, Stuttgart, Leipzig, puis Varsovie et en mai 1940 à Luxemburg. Ceci permettait de faire croire au déplacement de l'émetteur et donc de renforcer sa crédibilité d'émetteur clandestin communiste en France. Les speakers recrutés pour cette « radio noire » ne sont pas connus. Seul un certain monsieur Thony, un acteur ayant grandit en France, est nommé dans un courrier d'Otto Abetz le 30 janvier $1940^{49}$.

Alors que la première radio secrète allemande naît en décembre 1939, la première « radio noire » française (Der deutsche Freiheitssender) à destination de l'Allemagne émet dès le jour de la déclaration de guerre. S'il a été impossible d'en trouver la moindre trace dans les archives françaises, c'est dans les archives militaires allemandes que l'on trouve la preuve de leur existence et un détail de leurs actions. Der Deutsche Freiheitsender émet deux fois par soir, à $20 \mathrm{~h} 30 / 21 \mathrm{~h}$ et $22 \mathrm{~h}^{50}$, sur différentes longueurs d'ondes tout en changeant d'émetteurs. Sous l'impulsion de l'IEE de Strasbourg, évacué à Salins dans le Jura, des émissions en langue allemande sont radiodiffusées à partir du 9 octobre $1939^{51}$. Il est indiqué dans une note datée du 9 octobre :

« À partir du 11 octobre, [seront émises] deux émissions par jour, une avant midi, l'autre vers 16 heures, sans préjudice d'émissions d'urgence sous trois heures de préavis maximum, toutes les fois que le caractère ou l'énoncé d'émissions de langue française ou de langue allemande de la radio du Reich exigera une réplique immédiate ${ }^{52}$.

Ces émissions dont la tactique est de faire varier le style et la longueur des textes ont pour mission de «répondre à vue et en s'appuyant sur une information de base extraite d'une très vaste documentation de politique extérieure constamment à portée de notre main, aux imputations ou aux agressions les plus diverses de la radio ennemie $\gg^{53}$.

Au début, ces émissions ne sont ni soumises au contrôle du Commissariat ni à celui du Grand Quartier Général, puis, le 7 novembre 1939, les deux institutions estiment « que les émissions radiophoniques de Salins doivent être, comme toutes les autres émissions de ce genre, soumises à un contrôle préalable au CGI en accord avec le GQG $\gg^{54}$. Il y a par ailleurs une réelle volonté d'adaptation de la part des différentes institutions. Au mois de janvier 1940, le GQG, conscient des actions de brouillage des ondes françaises par les autorités allemandes, propose de «changer fréquemment la longueur d'onde des émissions $\gg^{55}$. D'autre part, le 10 avril, il conseille de diffuser les émissions françaises en langue allemande sur les longueurs d'onde les plus voisines de celles captées par les auditeurs allemands, «les émissions françaises en langue allemande ainsi diffusées [...] auraient plus de chances, semble-t-il, d'être entendues par eux sans crainte de représailles ou de sanctions $\gg^{56}$. Une autre tentative d'adaptation est celle visant plus particulièrement les militaires allemands de l'autre côté du Rhin. En dehors de leur récepteur de radio

48 Ibidem, p. 44.

49 Ibidem, p. 45.

50 BArch-MA, RW4/24.

$51 \mathrm{AD}$ Bas-Rhin, «Emissions radiophoniques de langue française et de langue allemande des 9 et 10 octobre $1939 », 11.10 .1939$.

52 Idem.

53 Idem.

54 AN F/41/979, « Note du G.Q.G. pour le Commissariat Général à l’Information », 7.11.1939.

55 AN F/41/21-22, « Radio allemande », janvier 1940.

56 AN F/41/21-22, « Emissions de Radio en Allemagne », 10.4.1940. 
militaire, ceux-ci disposent majoritairement de ces appareils récepteurs peu coûteux, les Volksempfänger («récepteur du peuple»), mais dont la capacité reste limitée. Le GQG, en ayant été informé, demande à ce que soit «déterminé exactement quelles sont les stations d'émissions qui peuvent être entendues par les Volksempfänger et de spécialiser ces stations le plus rapidement possible dans la diffusion de nos émissions de propagande en langue allemande $»^{57}$. Ainsi, à travers toutes ces initiatives et tentatives d'adaptation, il est possible de constater que les autorités françaises chargées de la propagande n'ont pas déconsidérer l'importance de la propagande de guerre et ont été au contraire dynamiques dans ce domaine.

\section{THEMATIQUES, TECHNIQUES ET RECEPTION DES PROPAGANDES RADIOPHONIQUES}

\section{La propagande allemande et sa réception en France}

Tout comme la radio du «traître Ferdonnet ${ }^{58}$, les programmes de La Voix de la Paix, et de Radio Humanité rejoignent la campagne principale de propagande allemande; la critique de l'alliance franco-britannique. Celle-ci constitue est présente dans 56,5\% $\%$ des tracts allemands envoyés en France et constitue $70 \%$ de la propagande radiophonique allemande en langue française. D'une manière générale, ces arguments de Spaltungspropaganda ou Zersetzungspropaganda ${ }^{61}$ («propagande de séparation / de dissociation ») ont pour objectif de briser l'alliance franco-britannique. Déjà employé lors de la Première Guerre mondiale, le thème de l'opposition entre Français et Anglais est loin d'être novateur. Un chercheur de l'IEE rappelle en octobre 1939 :

«On, ne saurait trop insister sur le rapprochement qu'il convient de faire avec la guerre de 1914-1918. À ce moment déjà l'Angleterre avait pris une décision qui n'était pas attendue [la déclaration de guerre] et qui par là même suscita l'indignation de l'Allemagne impériale. ${ }^{62}$

Certaines affirmations valables pour la Première Guerre mondiale le sont également pour la période de la «drôle de guerre ». Les historiens Krumeich et Becker affirment que le premier conflit mondial, «plus qu'un conflit franco-allemand, apparut [aux Allemands] comme un conflit avec l'Angleterre, dont la France n'était qu'un bras armé ${ }^{63}$. Lorsque la «drôle de guerre » se met en place, ce discours anti-britannique s'inscrit alors dans une certaine continuité de la propagande allemande. Dès le 3 septembre 1939, les émissions en langue française de Radio Stuttgart retracent le déroulement de la déclaration de guerre, présentant l'Angleterre comme responsable de la guerre :

«Le gouvernement britannique a, en première ligne, figuré parmi ceux qui, par leur attitude intransigeante, ont fait échouer toute révision pratique [du traité de Versailles] sans l'intervention du gouvernement britannique on aurait certainement pu trouver une solution raisonnable et juste pour les deux parties ${ }^{64}$

57 AN F/41/21-22, « Emissions de Radio en Allemagne », 24.4.1940.

58 Jean-Louis Crémieux-Brilhac, op.cit., p.375.

59 Calcul basé à partir du recueil de Kirchner Klaus, Flugblatt-Propaganda im 2. Weltkrieg Europa, t. 2 , Erlangen, Verlag für Zeitgeschichtliche Dokumente und Curiosa, 1982.

60 Chiffre donné dans Jean-Louis Crémieux Brilhac, op.cit.

61 BArch-MA, RW4/241, 30.9.1939.

62 A.D. Bas-Rhin, 4.11.1939

63 Jean-Jacques Becker, Gerd Krumeich, La Grande Guerre: Une histoire franco-allemande, Paris, Tallandier, 2008, p. 9.

64 AN F43/95 
Ainsi, lorsque le speaker de Radio Stuttgart, André Obrecht, affirme que «le gouvernement britannique porte la responsabilité pour tout le malheur et toutes les souffrances qui assaillent maintenant et qui vont assaillir de nombreux peuples ${ }^{65}$, il ne fait que prolonger cet argument. Cette propagande s'amplifie au fur et à mesure des mois de guerre, devenant de plus en plus virulente. Parallèlement à la responsabilité de l'Angleterre dans le déclenchement de la guerre, l'idée de l'instrumentalisation par Churchill des soldats français comme «bouclier» pour protéger les Tommies prend de l'ampleur. Radio Stuttgart diffuse à partir du 23 septembre 1939 ce slogan : «Auditeurs français, l'Angleterre fournit les machines alors que la France fournit les poitrines ${ }^{66}$ qui devient par la suite le leitmotiv de la radio allemande.

À cet argumentaire visant la désolidarisation de la France d'avec l'Angleterre s'ajoute une spécificité pour les «radios noires »; le renforcement des dissensions politiques internes. En particulier valable pour Radio Humanité, les sujets font apparaitre la critique des « 200 familles » portée par le Parti Communiste d'avant guerre ${ }^{67}$ tentant ainsi de briser la nouvelle tentative d' «Union sacrée » déjà titubante. Des hommes politiques tels $\mathrm{M}$. Sarraut, ministre de l'Intérieur sous le gouvernement Daladier, M. Daurty, ministre de l'Armement y sont interpellés et ridiculisés ${ }^{68}$. De plus, alors que Radio Stuttgart tente de créer un rapprochement avec Hitler, le présentant comme un homme n'ayant aucune intention belliqueuse envers la France, les « radios noires » critiquent abondamment Hitler et sa politique. Ainsi, l'image d'Attila - répandue en France à cette époque pour désigner Hitler - est utilisée dans les discours radiodiffusés de Radio Humanité, renforçant ainsi l'illusion de l'émission. Par ailleurs, que l'information vienne d'une « radio blanche » ou d'une «radio noire », l'objectif principal reste le même. Il s'agit de déstabiliser psychologiquement la population et les troupes de l'adversaire en les inondant d'informations qui les poussent à remettre en cause la véracité des informations données par leur gouvernement.

La répétition abusive du slogan anti-britannique correspond à l'une des directives de Goebbels concernant la propagande, énoncée lors d'une conférence de son ministère ${ }^{69}$. Un rapport de l'IEE daté du 14 avril 1940 témoigne que dans les émissions allemandes en langue française il «s'agit uniquement de propagande, d'enfoncer les idées simples, fréquemment répétées, dans l'esprit des auditeurs $\gg^{70}$. Ils constatent également que la propagande radiophonique allemande fait preuve de vigueur et de rapidité et que l'impact des «slogans » ne doit pas être sous-estimé ${ }^{71}$. Jean-Louis Crémieux-Brilhac, qui a analysé les discours de Radio Stuttgart, affirme :

«Elle mène à la guerre anglaise une campagne acharnée, à toute occasion et parfois à tort et à travers, le plus souvent avec lourdeur [et que] quand radio Stuttgart s'essaie à faire de l'esprit, c'est avec lourdeur et vulgarité $\gg^{72}$

De plus, les émissions d'information en dehors de leur récepteur de radio militaire procèdent souvent par ruse. Des informations de propagande sont en effet insérées entre deux bandes musicales, souvent de la musique populaire française, afin d'attirer l'auditeur

65 AD Bas-Rhin, 98AL649, 31.9.1939.

66 AN F43/95-133.

67 Pendant l'Entre-deux-guerres, le Parti Communiste dénonçait fortement les « 200 familles » les plus riches de France, qui auraient été, selon lui, responsables du malheur du peuple français.

68 BArch-MA, RWA/312a, « Auszug aus Bericht des spanischen Mil. Att. In Paris vom 10.1.1940 ».

69 Idem.

70 AD Bas-Rhin, 98AL658, «L'écoute de la radio en Alsace », 14.4.1940.

71 AD Bas-Rhin, 98AL649, « Les aspects de la propagande radiophonique allemande », 17.9.1939.

72 Jean-Louis Crémieux-Brilhac, op.cit., p. 382. 
français qui se retrouve alors en train d'écouter des informations de Radio Stuttgart, comme en témoigne Maitre Heitz :

«De Stuttgart, de Francfort, de Munich on émet, presque sans interruption, des programmes alléchants comme, par exemple, des airs populaires, de grands opéras, ou, comme cet aprèsmidi (16 octobre), un programme entier de musique française, entrecoupé de nouvelles tendancieuses en français $»^{73}$

Comme nous n'avons pas eu accès aux bandes-son des émissions, les informations relatives au ton employé proviennent de rapports français. Le vocabulaire employé est virulent face aux Anglais. À l'occasion du discours de M. Chamberlain, le 14 octobre 1939, un torrent d'injures se déverse sur les membres du gouvernement anglais :

«On se demande si ce vieillard chancelant et qui est dépourvu de tout raisonnement logique veut lancer trois nations dans une aventure aussi criminelle. La presse de tous les pays est unanime à condamner la fureur belliciste du Premier anglais $\gg^{74}$

Un rapport de l'IEE affirme que le ton des émissions allemandes est «extrêmement ironique et vulgaire ${ }^{75}$ et de manière générale «plutôt agressif que mou ${ }^{76}$. La sémantique employée dans les émissions à l'égard des Anglais, se retrouve également dans les tracts. Les Anglais sont souvent liés aux champs lexicaux de la mort et de la traîtrise, alors que les Français sont présentés en victime. Des slogans tels que : "C'est avec le sang de ses alliés que l'Angleterre a toujours écrit son histoire ${ }^{77}$, ou encore $" C^{\prime}$ 'est pour les intérêts de l'Angleterre, pour la Banque anglaise, que la jeunesse de France doit verser son sang ! ${ }^{78}$ sont ainsi très agressifs et usent d'un vocabulaire imagé très fort. Cette violence à l'encontre des Anglais se retrouve également dans les images et les dessins présents dans les tracts allemands qui représentent souvent des soldats français morts ou blessés, amputés, encore saignants, et contrastant avec la bonne humeur des Anglais ${ }^{79}$. De telles paroles et images font appel à l'émotivité du lecteur ou de l'auditeur, rejoignant ainsi l'un des principes de la propagande de Goebbels qui ne désire pas combattre par un raisonnement intellectuel, mais par l'éveil d'émotions, par l'intermédiaire de phrases et d'images choquantes. Cependant, les autorités allemandes et françaises font remarquer des faiblesses de style et de forme de la propagande allemande. Les Français relèvent, par le biais de l'IEE de Strasbourg, que la propagande radiophonique allemande est, du moins au début du conflit, souvent exercée par «d'authentiques Teutons, dont l'accent ne trompe pas ${ }^{80}$. Un sketch radiodiffusé est particulièrement visé : émise maintes fois sur les ondes de Radio Stuttgart, la courte scène théâtrale «Fachoda» fait parler Napoléon III et Ferdinand de Lesseps dont l'«accent tudesque (qui) est irrésistible et (qui) ruine définitivement tout l'effort des émissions françaises de la propagande allemande auprès de ceux qui ont entendu cette petite comédie ${ }^{81}$. Sans aller jusqu'à dire que cette pièce « ruine définitivement » l'action de propagande allemande, comme l'affirme à l'époque un employé de l'IEE, on peut dire qu'elle compromet la crédibilité générale de l'émission,

73 AD Bas-Rhin, 98AL450, «Vue sur la propagande et les problèmes alsaciens », 25.10.1939.

74 AD Bas-Rhin, 98AL651, « Deux mois de propagande radiophonique allemande », 4..11.1939.

75 AD Bas-Rhin, 98AL655, «Une émission de langue française de la radio allemande contre M. Winston Churchill », 30.01.1940.

76 AD Bas-Rhin, 98AL649, « Remarques sur la propagande radiophonique », 23.09.1939.

77 Klaus Kirchner, op.cit., pp. 72-73.

78 Ibid., p. 62. «Soldats français ! Pour qui combattez vous ?»

79 Klaus Kirchner, op.cit., p. 111.

80 AD Bas-Rhin, 98AL651, « Deux mois de propagande radiophonique allemande », 4..11.1939.

81 Idem. 
sans toutefois avoir un caractère irréversible. Cette remarque sur l'accent des speakers est aussi relevée par les autorités allemandes. Dans une lettre datée du 18 octobre 1939, le docteur H. Spranger rapporte au ministère de la guerre qu'une émission de la radio allemande diffusait une émission d'information en langue française avec un accent français « on ne peut plus mauvais ${ }^{82}$. De plus, au fil des mois, la propagande anti-britannique allemande s'essouffle à cause des trop nombreuses répétitions qui, aux yeux de certains Français, expriment «une réalisation médiocre, dont la pauvreté de l'imagination, l'indigence de l'invention, le peu de conviction des affirmations sont responsables ${ }^{83}$. De même, ce «principe heureux de la propagande allemande ${ }^{84}$ qu'est la répétition, engendre quelques déconvenues :

«Pour exemple, lorsque la radio allemande annonce dix fois de suite qu'un commandant de sous marin allemand a donné une bouteille de gin à l'équipage d'un navire qu'il avait coulé, ce détail finit rapidement par devenir suspect pour tout esprit un peu critique $»^{85}$

La même constatation peut être faite sur le ton employé par le speaker de Radio Stuttgart, usant «d'appels larmoyants et romantiques » qui à force de répétition «durant de longs jours», perdent de leur effet des premiers instants ${ }^{86}$.

On comprend donc que la réception en France de ces émissions pendant la «drôle de guerre » est plutôt mitigée et qu'on ne peut parler d'un réel impact sur la population française de la campagne anti-britannique qui pourtant est au centre de la propagande allemande. Même si dans certains milieux, déjà anglophobes avant la guerre, cette campagne trouve une résonance. Face à ce flux d'informations constant de la propagande allemande, les autorités françaises ne sont pas en reste.

\section{Les arguments français et leur résonance en Allemagne}

Face à la propagande offensive allemande, les historiens évoquent une simple réponse de la part des autorités françaises, allant parfois jusqu'à affirmer: «contre les tracts allemands, contre Radio Stuttgart, que faisons nous ? Rien ou presque rien ${ }^{87}$. Cependant, la propagande française en langue allemande (par tract ou par radio) n'est pas inactive et ne se cantonne pas à une simple défense. D'ailleurs, les risques d'une passivité dans ce domaine ont été dénoncés le 30 septembre 1939 par l'IEE de Strasbourg et transmis aux autorités responsables, affirmant que la propagande française «doit s'attacher à ne pas être purement fondée sur une idée de défensive ${ }^{88}$. Toutefois, cette propagande défensive existe, tout comme la propagande défensive allemande. Il s'agit pour la France de contrer l'argument principal de l'ennemi, qui vise, comme nous l'avons vu, à séparer la France de son allié. Il s'agit par exemple de montrer par tract des statistiques sur la flotte anglaise qui se bat aux côtés des Français ou bien encore de remémorer le lien d'alliance entre la France et la Grande-Bretagne pendant la Première Guerre mondiale ${ }^{89}$. Par exemple, lors de la journée du 11 novembre 1939, jour de commémoration de l'armistice de 1918, les émissions françaises en langue allemande insistent sur la longue tradition de l'investissement des Anglais et la participation toujours plus accrue du contingent anglais

82 BArch-MA, RW4/283, 18.9.1939.

83 AD Bas-Rhin, 98AL651, « Deux mois de propagande radiophonique allemande », 4..11.1939.

84 Idem.

85 Idem.

86 Idem.

87 Henri Amouroux, op.cit., p. 216.

88 AD Bas-Rhin, 98AL649, « Propagande et contre propagande radiophonique de guerre », 30.09.1939.

89 Klaus Kirchner, op.cit. 
dans le conflit - notamment avec l'agrandissement du British Expeditionary Force prévu jusqu'au printemps ${ }^{90}$.

Ceci dit, la propagande française ne se limite pas à la simple contre-attaque des arguments allemands. En effet, parmi les différents arguments de persuasion des émissions françaises en langue allemande, se dessine une ligne stratégique majeure : la tentative de séparation entre le peuple allemand et Hitler. Cette ligne directrice est clairement visible. Le rejet de la responsabilité de la guerre sur les dirigeants allemands, et notamment sur Hitler, en constitue la base. De plus, les autorités françaises désirent marquer une forte distinction entre le peuple allemand et la NSDAP, SS, SA, hauts-dirigeants et Hitler afin de créer des tensions entre eux, et si possible, de mener au renversement du régime hitlérien. Ainsi, sur les ondes de Radio Strasbourg on peut entendre : " les SS feront leur devoir à l'arrière jusqu'au dernier soldat du front allemand $»^{91}$, mimant ainsi le slogan antibritannique allemand.

Visant l'exacerbation de tensions entre le peuple et les dirigeants nationaux-socialistes, les émissions françaises de langue allemande rapportent également des événements de rébellion à l'intérieur du pays, incitant les populations à continuer et intensifier la révolte. Dans une émission du 27 octobre 1939, le speaker des émissions du soir, Pascal Copeau, affirme que "l'unité du Reich est menacée. Dans le sud de l'Allemagne, le courant Los von Berlin! ( «Hors de Berlin!»), se fait désormais remarquer. ${ }^{92}$. Il insiste également sur une supposée «position fragile d'Hitler dans son gouvernement $»^{93}$ et incite clairement le peuple allemand à la révolte dans son émission du soir, le dimanche 23 octobre 1939 :

\footnotetext{
«Il nous intéresse uniquement ce que vous, chers auditeurs, vous pensez de ces déchaînements. Vous ne pouvez attendre désormais de vos ministres que vociférations et hurlements... Le pouvoir que vous avez donné, vous pouvez également le reprendre. Tous les Berlinois pourraient, aujourd'hui encore, aller sur la Wilhelmplatz et lorsque les meurtriers de la Pologne arrivent, leur crier: 'Nous ne voulons pas de la Tchécoslovaquie, nous ne voulons pas de la Pologne, nous voulons la paix’ ${ }^{94}$
}

Le jour de Noël, le speaker français incite même les Allemands à penser aux victimes de ce régime «cruel», qui ont été exécutées et torturées car elles «pensaient différemment d'Hitler $»^{95}$. Il défend ainsi la liberté d'expression, sans en dire le nom, et avec elle les valeurs et principes de la démocratie. La difficulté pour la radio est néanmoins de toucher un maximum de personnes aux conditions de vie très contrastées : Radio Strasbourg doit en effet s'adresser à la fois à l'ouvrier, au soldat allemand, mais également à la femme au foyer. Cette dernière cible est particulièrement interpellée par Françoise Rosay, une comédienne française, lors de «causeries » pendant lesquelles elle leur tient un discours émancipateur ${ }^{96}$. Ces tentatives, qui consistent à dresser les Allemands contre leurs dirigeants, s'enrichissent des événements de l'actualité comme par exemple l'attentat de Munich qui a été «exploité par la propagande ennemie [française] comme symbole d'oppositions massives ${ }^{97}$. Puis, avec l'augmentation des restrictions, la mise en place des coupons d'alimentation et vestimentaires, les attaques visant les dirigeants allemands ne cessent de croître. Parallèlement à cette Spaltungspropaganda qui est présente dans $75 \%$ des tracts français en langue allemande, une autre thématique importante se répand en

90 BArch-MA, RW4/242, « Lagebericht der Rundfunk », 30.11.1939.

91 BArch-MA, RW4/241, 24.09.1939.

92 BArch-MA, RW4/242, 27.10.1939.

93 Idem.

94 Idem.

95 BArch-MA, RW4/242, « Lagebericht der Rundfunk », 30.12.1939.

96 BArch-MA, RW4/241, 26.11.1939.

97 BArch-MA, RW4/363, « Abwehr feindlicher Propaganda », 6.12.1939. 
Allemagne : la remise en cause de l'union entre Staline et Hitler. Dans un Lagebericht der Rundfunk le constat est que :

«Jusqu'au 27 septembre 1939 la propagande des pays ennemis était peu sûre, prudente et retenue. Mais après le discours de Ribbentrop à Moscou, elle s'est transformée, changeant le discours en un ton agressif disant qu'ils combattraient jusqu'à l'anéantissement du régime hitlérien $»^{98}$

À partir de cette date, les discours visant à remettre en cause l'alliance germano-soviétique se multiplient. Du 21 au 26 octobre 1939, les speakers de Radio Strasbourg insistent sur l'« échec de la politique russe d'Hitler car Staline refuse de fournir toute aide militaire contre les forces occidentales. $\gg 99$.

Le style des émissions radiophoniques françaises n'est pas fondamentalement différent de celui des émissions allemandes. Elles privilégient également les phrases courtes, comme par exemple dans cette émission particulièrement virulente et dont le style peut rappeler celui des speakers allemands :

\begin{abstract}
«Vous tous voulez éliminer la domination des caïds des Nazis !, À bas les chiens à la recherche de sang et les criminels !, Dehors Hitler l'idiot qui souffre de mégalomanie !, [...] La pure vérité vient des stations radiophoniques étrangères, [...] À bas Hitler le dégénéré ! À bas Goebbels l'estropié !, À bas Rosenberg, l’opposant à la chrétienté ! ${ }^{100}$
\end{abstract}

Au-delà de la simple technique du slogan, on se rend bien compte du caractère incisif et ciblé des propos diffusés par la propagande française et ce relativement tôt. En effet, les Allemands constatent un accroissement de l'agressivité de l'idéologie et de la tactique ennemies, accompagné de l'accentuation du ton virulent ${ }^{101}$. Le rapport sur la propagande radiophonique française indique qu'un discours de haine se répand par la radio à travers des moqueries et des insultes ${ }^{102}$. Il est aussi rapporté qu'il «n'y a plus de différence désormais entre la radio française et anglaise. Les deux voix coïncident parfaitement dans la méthode et le ton ${ }^{103}$. Ce rapprochement entre les émissions françaises et anglaises n'est pas un cas isolé. Un mois plus tard, le même constat est fait : "Les propagandes radiophoniques françaises et anglaises sont à présent dans leur tactique et leur ton tellement similaires, que les émissions de Strasbourg et de Daventry coïncident presque parfaitement dans leur forme, contenu, et construction ${ }^{104}$. Il semble ainsi aux autorités allemandes que les deux alliés travaillent « main dans la main pour la combinaison de leurs affirmations et accusations $»{ }^{105}$. Venues de France, certaines émissions sont dynamiques et contiennent des propos corrosifs à l'encontre du régime et des dirigeants nationauxsocialistes. Les émissions du célèbre réalisateur allemand Max Ophüls qui a fui le régime nazi et s'est réfugié à Paris, sont diffusées au début de l'année 1940 et sont composées de pièces radiophoniques ou chansons ${ }^{106}$ dans lesquelles Hitler est la cible d'invectives virulentes et où l'ironie et la moquerie ont une place prédominante ${ }^{107}$.

98 Traduction de l'auteur de BArch-MA, RW4/241, « Lagebericht der Rundfunk », 27.09.1939.

99 BArch-MA, RW4/242, 27.10.1939.

100 Traduction de l'auteur de BArch-MA, RW4/242, 1940.

101 BArch-MA, RW4/242, « Lagebericht der Rundfunk », 16.10.1939.

102 BArch-MA, RW4/242, 1940

103 Idem.

104 BArch-MA, RW4/241, 7.11.1939.

105 Idem.

106 Helmut G. Asper, « Ophuls à la radio », 1895, Mille huit cent quatre-vingt-quinze, http://1895.revues.org/212 Site visité en mars 2013. Deux chansons « Dors, Hitler, dors !», « La Nouvelle Base de Wessel » et deux pièces radiophoniques, « Kinderspiele » et « Les Sept Crimes d'Adolf Hitler ». 107 Idem. 
Le ton des émissions françaises en langue allemande s'agrémente en effet principalement d'ironie, de parodie et d'humour ${ }^{108}$. Pascal Copeau est d'ailleurs connu en Allemagne pour tenir des propos particulièrement ironiques envers la radio allemande. Dans l'une de ses émissions de 23h15, il se moque ouvertement de la propagande allemande :

« Nous savions que le métier de journaliste en Allemagne demandait une très grande capacité d'imagination, mais quelques connaissances sont tout de même essentielles. [...] Un proverbe français dit «Le ridicule tue ». Malheureusement il ne semble pas que ce soit le cas en Allemagne chez les petites gens de la propagande allemande. (Strasbourg, 2.10.) ${ }^{109}$

Bien que la Troisième République affirme à maintes reprises que la propagande allemande est mensongère et que les informations venues de France sont, quant à elles, véridiques, l'analyse des faits donne une vision bien différente et montre que le mensonge n'est pas l'apanage du régime nazi. Dans les émissions françaises en langue allemande, des histoires fausses se font entendre, relevées ensuite par les autorités allemandes. Le 6 décembre 1939 par exemple, un fait divers est raconté par le speaker français. Il met en scène une femme allemande qui va chez l'épicier et qui se retrouve face à des policiers. Ceux-ci se croyant insultés, arrêtent toute la clientèle féminine du magasin, soit onze femmes ${ }^{110}$. Cette affaire, après une enquête discrète de la part des autorités allemandes, est identifiée comme étant le fruit de l'imagination des autorités françaises. Cependant, pour les propagandistes français, l'essentiel est que ce fait divers soit vraisemblable. En citant des numéros de téléphone, des noms de rues, des noms de personnes, le speaker rend son histoire réelle pour l'auditeur. Alors que la citation des pages de Mein Kampf se doit de donner les références exactes car tout le monde possède l'ouvrage, les informations données pour cette affaire ne peuvent être vérifiées par un simple citoyen et seule l'apparence de vérité compte ${ }^{111}$. Ici l'idéal d'une république démocratique diffusant des informations véridiques et usant de finesse dans sa propagande se voit fortement altéré. Identifier la propagande venant de France comme une propagande caractéristique d'un état démocratique n'a alors que peu de sens. Lorsque l'on parle de la forme, du style et du ton de la propagande française destinée à l'étranger, il n'y a que peu de différence avec ceux utilisés par l'Allemagne en direction de la France.

L'étude de la propagande radiophonique française et allemande permet de mettre en évidence le rapport de force entre les deux propagandes. Au niveau institutionnel, des défaillances, parfois similaires, sont présentes, notamment en ce qui concerne les tentatives d'ingérence des différents organes chargés des affaires de propagande comme l'Armée, le ministère des Affaires étrangères et l'institution spécialement dévolue à la propagande (Commissariat général à l'Information en France et Ministère de la Propagande en Allemagne). Il ressort également de la comparaison de ces deux fonctionnements que la propagande radiophonique met en scène de nombreux acteurs issus de différents services et aux visions parfois divergentes. En France ou en Allemagne, les institutions ont recours aux mêmes procédés et créent des émissions radiophoniques «blanches » et «noires » répondant à des objectifs spécifiques. Les dirigeants de la propagande radiophonique font également appel aux services de locuteurs natifs afin de rendre leur propagande plus crédible. De nombreuses similitudes apparaissent ainsi dans le fonctionnement des émissions radios dans la langue de l'ennemi.

108 BArch-MA, RW4/363, «Abwehr feindlicher Propaganda », 29.01.1940.

109 BArch-MA, RW4/241, 2.10.1939.

110 BArch-MA, RW4/363, «Abwehr feindliche Propaganda », 6.12.1939.

111 Idem. 
On comprend alors la nécessité de réévaluer le constat des historiens sur la propagande française durant la «drôle de guerre ». Alors qu'ils lui attribuent une action faible et cantonnée à un cadre défensif duquel elle ne sort que très rarement, elle est en réalité dynamique, claire, cohérente et rusée, à la fois en se défendant des attaques ennemies et en faisant preuve d'initiative. Chacun des deux Etats belligérants a recours aux mêmes techniques et adapte ses thématiques à la situation politique et sociale du pays adverse. La propagande allemande n'est que peu teintée d'antisémitisme et de pangermanisme. Elle se préoccupe davantage de dénoncer la responsabilité anglaise dans le déclenchement de la guerre. Du côté français, on critique l'hitlérisme et l'oppression des dirigeants nazis, sans pour autant prôner ouvertement des valeurs démocratiques et républicaines. De plus, dans la forme et le vocabulaire employés, on trouve de nombreuses similitudes : la propagande allemande envers l'ennemi ne peut être qualifiée de plus mensongère ou plus virulente que la propagande française en langue allemande. Ainsi, l'étude de la propagande radiophonique à destination de l'ennemi entre la France et l'Allemagne pendant la « drôle de guerre » semble montrer que dans le domaine de la propagande extérieure, les méthodes d'un état démocratique qu'est la Troisième République ne se différencient qu'en de très légers points de celles d'un état totalitaire, le Troisième Reich. et que des similitudes inhérentes à la période sont à relevées. 
ANNEXES

Annexe 1:

Organisation de l'appareil radiophonique de propagande allemand en décembre 1939.

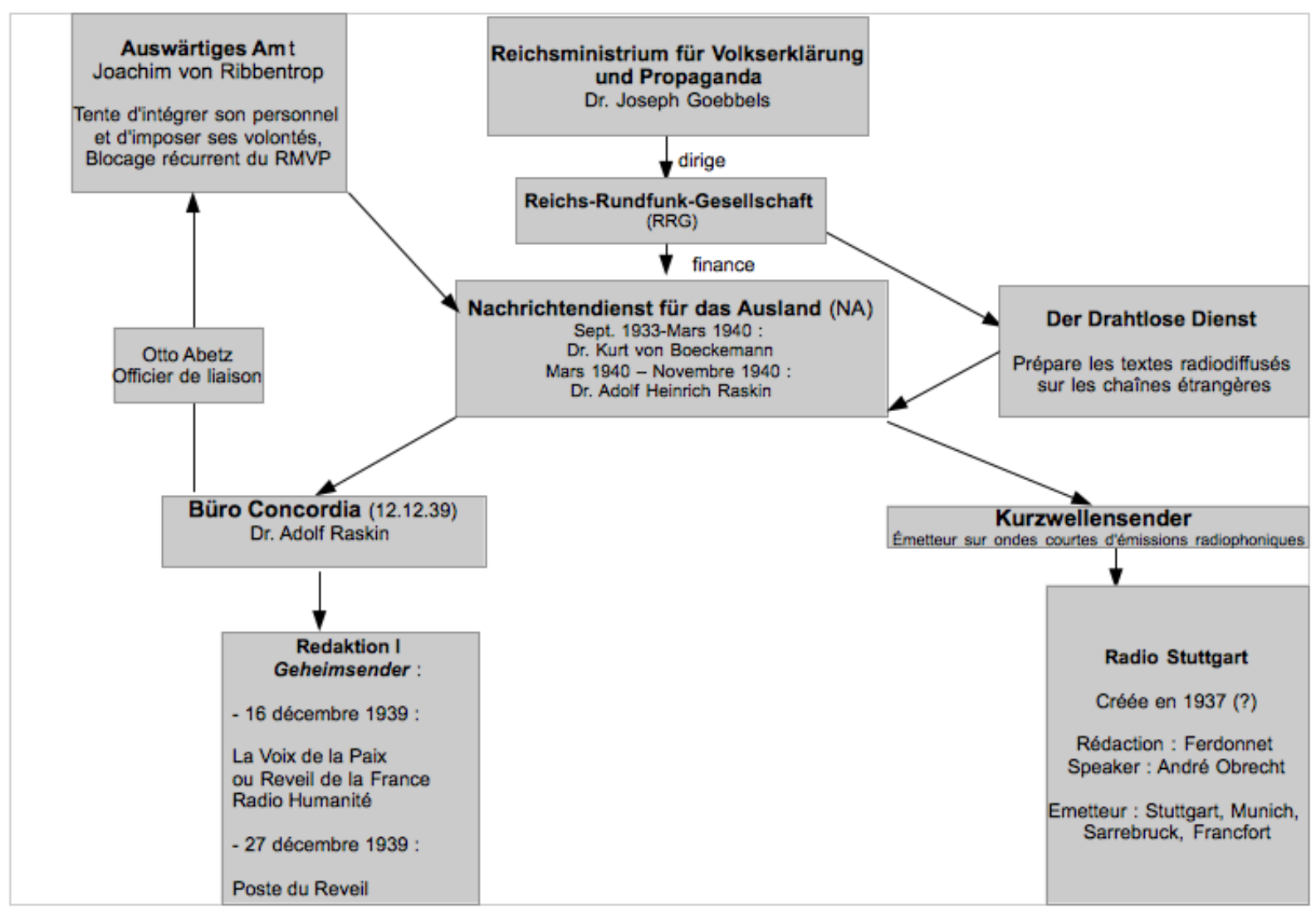


Annexe 2:

Organisation de l'appareil de propagande radiophonique français en septembre 1939

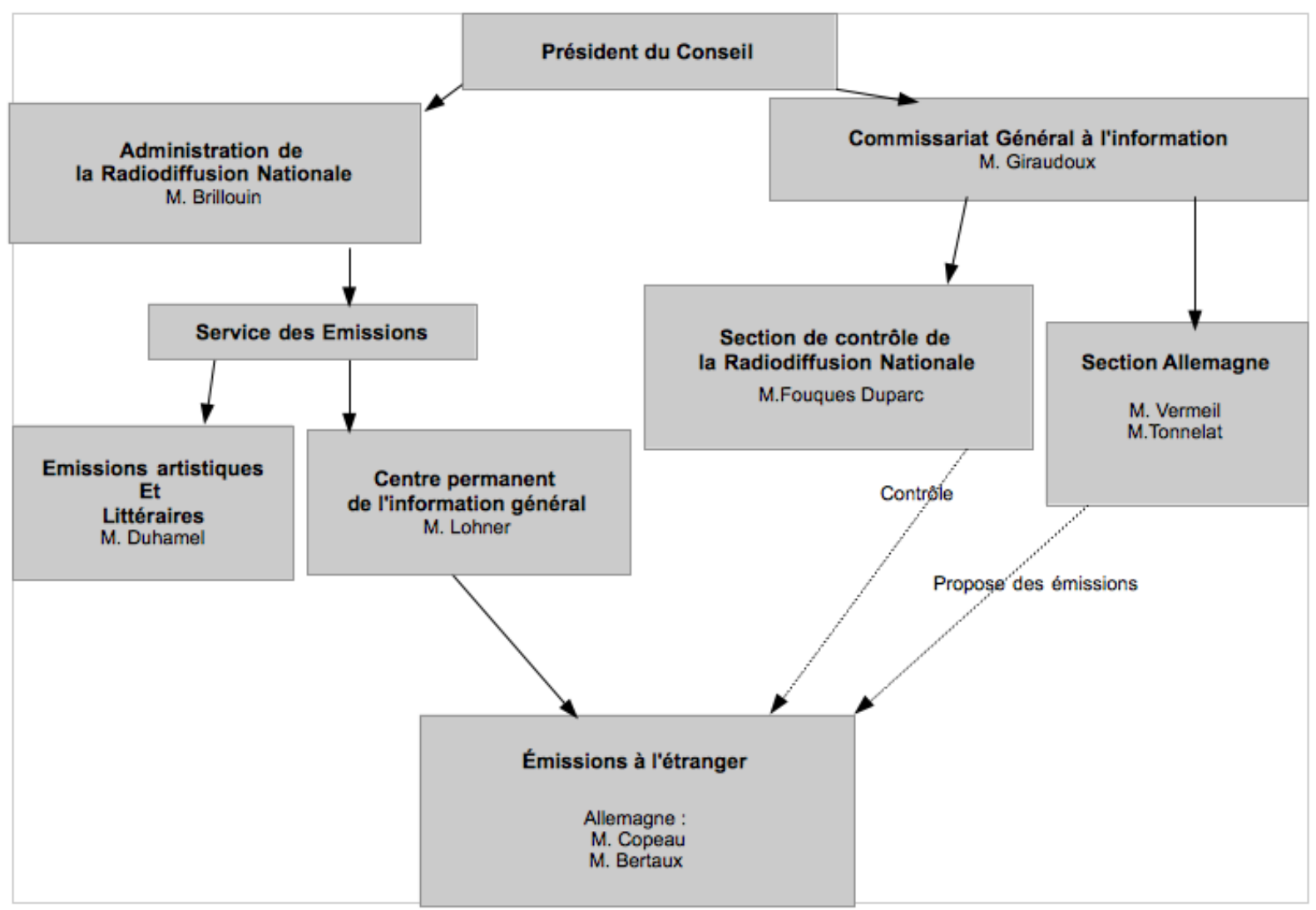

Sources : Organigramme réalisé à partir des fonds F/41/13 à 25 des Archives Nationales de Paris.

Notice Bio-bibliographique :

Maude Fagot est collaboratrice scientifique à l'université de la Sarre (Allemagne) et travaille actuellement au sein d'un projet franco-allemand sur les évacuations de la région frontalière francogermanique pendant la Seconde Guerre mondiale. En tant que doctorante en cotutelle sous la direction des professeurs Olivier Forcade de l'Université Paris-Sorbonne et Johannes Großmann de l'Université de Tübingen (Allemagne), elle oriente ses recherches vers l'étude de la médiatisation des évacuations dans les deux pays concernés.

\section{RESUMÉ :}

Alors que les opérations militaires entre la France et l'Allemagne n'ont pas encore pris l'ampleur qu'une déclaration de guerre est connue d'engendrer, un combat d'une autre nature fait rage. La guerre à laquelle se livre les deux pays se joue en effet dans le domaine de l'éther ; à travers les ondes radiophoniques. Les deux nations utilisent ce nouveau medium de masse qu'est la radiodiffusion pour atteindre la population, l'armée ennemie et tentent d'influencer son moral par le biais de mots, de slogans et d'arguments corrosifs. Alors que la supériorité de la propagande allemande est communément acceptée dans l'historiographie des deux pays, il est peu connu - mais n'en demeure pas moins vrai - que les autorités françaises se sont lancées dans cette bataille avec autant d'énergie. Les arguments de l'une et l'autre nation, bien que dissemblables, reposent sur des méthodes identiques de propagande, créant ainsi, dans le domaine de la propagande extérieure, des similitudes inhérentes à la période. 
Mots-clés :

Seconde Guerre mondiale - drôle de guerre - France-Allemagne - propagande - radiodiffusion

ABSTRACT :

In French as well as in German and British historiography, German propaganda during the Second World War is generally considered to have been more active and more effective than its French counterpart. This article calls this notion into question by looking at radio propaganda in France and Germany, and reevaluates the balance between the two. During the inter-war period, radio propaganda became increasingly organised, so that it may even be called 'centralised' by the time war broke out on 3 September 1939. Centralisation of propaganda leads to complex institutions, where rivalries arise between various services, as all of them are reluctant to cede prerogatives in the domain of information and propaganda to other institutions. However, both nations had established such an institution, i.e. a radio propaganda service to broadcast propaganda targeted at the enemy. In France, the Section germanique and in Germany the Büro Concordia organised radio broadcasts in the enemy's language. Two kinds of programmes - 'black' and 'white' - aimed at demoralising the enemy's civilian population and army in various ways. While 'black programmes' were disguised as national radio, pretending to be part of the broadcasting service of their listeners' country, 'white programmes' explicitly stated that their information came from the enemy. Thus, both civilians and soldiers in France and Germany would potentially be exposed to enemy propaganda, which was intended to convince them of their future defeat, and hence of the futility of carrying on with the war.

The approach the National Socialists took towards the French consisted mainly in virulent and aggressive anti-British propaganda. This campaign aimed at splitting the Franco-British alliance by attempting to lead the former to believe that they had been sent to the battlefield on their own and served British interests. Conversely, French propaganda targeted at Germany was more varied, yet two approaches figure prominently. The first approach taken was based on criticism of the SS, the SA, and Hitler, and called for a revolt of the German population against National Socialism, and Hitler in particular. The second line targeted the agreement Hitler and Stalin had reached in August 1939, by calling Hitler's authority into question. In both countries, France and Germany, radio propaganda was at work, employing the same methods, such as repetitions and slogans, and aimed at weakening the enemy's morale. Finally, there were no signs of particularities that could be attributed to French 'democracy' or German ‘totalitarianism'.

Keywords:

Second World War - Phoney War - France-Germany - propaganda - broadcasting 